\title{
THE GOHBERG LEMMA, COMPACTNESS, AND ESSENTIAL SPECTRUM OF OPERATORS ON COMPACT LIE GROUPS
}

\author{
By \\ Aparajita Dasgupta ${ }^{1}$ And Michael Ruzhansk ${ }^{2}$
}

\begin{abstract}
We prove a version of the Gohberg Lemma on compact Lie groups giving an estimate from below for the distance from a given operator to the set of compact operators. As a consequence, we obtain several results on bounds for the essential spectrum and a criterion for an operator to be compact. The conditions are given in terms of the matrix-valued symbols of operators.
\end{abstract}

\section{Introduction}

The original Gohberg Lemma was obtained during Gohberg's investigation of integral operators [Goh60], and its version on $\mathbb{T}^{1}=\{z \in \mathbb{C}:|z|=1\}$ was obtained recently by [MW10], with application to the spectral properties of operators; see [Mol11, Pir11]. Related questions have been also considered on manifolds; see, e.g., [See65, Sch88] and related papers.

In this paper, we establish the Gohberg Lemma on general compact Lie groups, using the matrix quantization of operators developed in [RT10, RT12]. In particular, we give estimates for the distance from a given operator to the set of compact operators, as well as for the essential spectrum of the operator in terms of some quantities associated to the matrix symbols. The results contain the corresponding results obtained in [Mol11, Pir11] on $\mathbb{T}^{1}$.

Matrix-valued symbols have been quite useful in other studies of compactness of operators in cases when conditions on the kernel are less effective, for example, by providing criteria for operators to belong to Schatten classes [DR13b] and for nuclearity in $L^{p}$-spaces [DR13a].

\footnotetext{
${ }^{1}$ The first author was supported by the Grace-Chisholm Young Fellowship of the London Mathematical Society.

${ }^{2}$ The second author was supported by the EPSRC Leadership Fellowship EP/G007233/1 and by EPSRC Grant EP/K039407/1. No new data was collected or generated during the course of the research.
} 
The structure of the paper is as follows. In Section 2, we briefly recall the necessary notions of Fourier analysis on compact Lie groups and of the matrix quantization of operators. In Section 3, we state our results. In Section 4, we prove the Gohberg Lemma (Theorem 3.1); and in Section 5, we give an application (Theorem 3.2).

\section{Fourier analysis and matrix symbols on compact Lie groups}

Let $G$ be a compact Lie group and $e$ its unit element. Let $\widehat{G}$ be the unitary dual of $G$, i.e., the set of equivalence classes $[\xi]$ of the continuous irreducible unitary representations $\xi: G \rightarrow \mathbb{C}^{d_{\xi} \times d_{\xi}}$ of dimension $d_{\xi}$. We define the Fourier coefficient at $\xi$ of $f \in C^{\infty}(G)$ by

$$
\widehat{f}(\xi):=\int_{G} f(x) \xi(x)^{*} d x \in \mathbb{C}^{d_{\xi} \times d_{\xi}},
$$

where the integral is (always) taken with respect to the Haar measure on $G$. The Fourier series becomes

$$
f(x)=\sum_{[\xi] \in \widehat{G}} d_{\xi} \operatorname{Tr}(\xi(x) \widehat{f}(\xi)),
$$

and Plancherel's identity takes the form

$$
\|f\|_{L^{2}(G)}=\left(\sum_{[\xi] \in \widehat{G}} d_{\xi}\|\widehat{f}(\xi)\|_{\mathrm{HS}}^{2}\right)^{1 / 2}=:\|\widehat{f}\|_{\ell^{2}(\widehat{G})} .
$$

We take (2.1) as the definition of the norm on the Hilbert space $\ell^{2}(\widehat{G})$. Recall that $\|\widehat{f}(\xi)\|_{\mathrm{HS}}^{2}=\operatorname{Tr}\left(\widehat{f}(\xi) \widehat{f}(\xi)^{*}\right)$ is the Hilbert-Schmidt norm of the matrix $\widehat{f}(\xi)$.

We define the matrix symbol of an operator $T: C^{\infty}(G) \rightarrow C^{\infty}(G)$ (or even $\left.T: C^{\infty}(G) \rightarrow \mathcal{D}^{\prime}(G)\right)$ by

$$
\sigma_{T}(x, \xi):=\xi(x)^{*}(T \xi)(x) \in \mathbb{C}^{d_{\xi} \times d_{\xi}},
$$

where $T \xi$ means $T$ applied to the matrix components of $\xi(x)$. It then follows that

$$
T f(x)=\sum_{[\xi] \in \widehat{G}} d_{\xi} \operatorname{Tr}\left(\xi(x) \sigma_{T}(x, \xi) \widehat{f}(\xi)\right) .
$$

The correspondence between operators and symbols is one-to-one, and we write $T_{\sigma}$ for the operator given by (2.2) corresponding to the symbol $\sigma(x, \xi)$. The quantization (2.2) has been studied extensively in [RT10, RT12], to which we refer for properties of the quantization and for the corresponding symbolic calculus. 
Recall that the matrix components of $\xi(x)$ are the eigenfunctions of the Laplacian (Casimir element) $\mathcal{L}$ on $G$ corresponding to one eigenvalue, which we denote by $\lambda_{\xi}^{2}$, i.e., $\mathcal{L} \xi(x)_{i j}=-\lambda_{\xi}^{2} \xi(x)_{i j}$ for all $1 \leq i, j \leq d_{\xi}$. We write $\langle\xi\rangle:=\left(1+\lambda_{\xi}^{2}\right)^{1 / 2}$.

Let $\Psi^{0}(G)$ be the usual class of operators that have symbols in Hörmander's class $S_{1,0}^{0}\left(\mathbb{R}^{n}\right)$ in every local coordinate system. Let $q_{1}, \ldots, q_{m} \in C^{\infty}(G)$ satisfy $q_{j}(e)=0, \nabla q_{j}(e) \neq 0$ for all $1 \leq j \leq m, e$ is the only common zero of the family $\left\{q_{j}\right\}_{j=1}^{m}$, and $\operatorname{rank}\left\{\nabla q_{1}(e), \cdots, \nabla q_{m}(e)\right\}=\operatorname{dim} G$. We call such a collection of functions strongly admissible. We then define $\Delta_{q_{j}} \widehat{f}(\xi):=\widehat{q_{j} f}(\xi)$ and the difference operators $\Delta_{\xi}^{\alpha}:=\Delta_{q_{1}}^{\alpha_{1}} \cdots \Delta_{q_{m}}^{\alpha_{m}}$. We refer to [RT10], and especially to [RTW10], for the analysis of such difference operators.

It was proved in [RTW10] that $T \in \Psi^{0}(G)$ is equivalent to the condition that the matrix-valued symbol $\sigma$ of $T$ satisfies

$$
\left\|\partial_{x}^{\beta} \Delta_{\xi}^{\alpha} \sigma(x, \xi)\right\|_{o p} \leq C_{\alpha \beta}\langle\xi\rangle^{-|\alpha|}
$$

for all $x \in G$ and $[\xi] \in \widehat{G}$ and for all $\alpha, \beta$, where $\|\cdot\|_{o p}$ stands for the operator norm of the matrix multiplication. It was also shown in [RTW10] that the operator $T \in \Psi^{0}(G)$ is elliptic if and only if its matrix symbol $\sigma(x, \xi)$ is invertible for all but finitely many $[\xi] \in \widehat{G}$ and for all such $\xi$,

$$
\left\|\sigma(x, \xi)^{-1}\right\|_{o p} \leq C
$$

for all $x \in G$.

\section{The Gohberg Lemma and applications}

We formulate a version of the Gohberg Lemma first for operators in the Hörmander class $\Psi^{0}$ in order to relate it to well-known theory and for application in Theorem 3.2. Later, in Remark 4.2, we note that the result remains valid for a much more general class of operators.

Let $\lambda_{1}(x, \xi), \lambda_{2}(x, \xi), \ldots, \lambda_{d_{\xi}}(x, \xi) \geq 0$ be the eigenvalues of $\sigma(x, \xi) \sigma(x, \xi)^{*}$ and define $\left\|\sigma(x, \xi) \sigma(x, \xi)^{*}\right\|_{\min }:=\min _{1 \leq i \leq d_{\xi}} \lambda_{i}(x, \xi)$.

Theorem 3.1 (Gohberg Lemma). Let $T_{\sigma} \in \Psi^{0}(G)$ and $\sigma(x, \xi)$ be the matrix symbol of $T_{\sigma}$. Then for all compact operators $K$ on $L^{2}(G)$,

$$
\left\|T_{\sigma}-K\right\|_{\mathscr{L}\left(L^{2}(G)\right)} \geq d_{\min },
$$

where

$$
d_{\min }:=\limsup _{\langle\xi\rangle \rightarrow \infty}\left\{\sup _{x \in G} \frac{\left\|\sigma(x, \xi) \sigma(x, \xi)^{*}\right\|_{\min }}{\|\sigma(x, \xi)\|_{o p}}\right\} .
$$


Note that $d_{\min }$ is well-defined. Indeed,

$$
\left\|\sigma(x, \xi) \sigma(x, \xi)^{*}\right\|_{\min } \leq\left\|\sigma(x, \xi) \sigma(x, \xi)^{*}\right\|_{o p} \leq\|\sigma(x, \xi)\|_{o p}^{2},
$$

which, in view of (2.3) with $\alpha=\beta=0$, implies

$$
d_{\min } \leq \limsup _{\langle\xi\rangle \rightarrow \infty}\left\{\sup _{x \in G}\|\sigma(x, \xi)\|_{o p}\right\}<\infty .
$$

We note again that the condition $T_{\sigma} \in \Psi^{0}(G)$ in Theorem 3.1 can be substantially relaxed; see Remark 4.2.

Before formulating our application of the Gohberg Lemma, let us first introduce some notation. Let $X$ be a complex Banach space and $A: X \rightarrow X$ be a closed linear operator with dense domain $D(A)$. The resolvent $\Phi(A)$ of $A$ is defined by

$$
\Phi(A)=\{\lambda \in \mathbb{C}: A-\lambda I \text { is bijective }\}
$$

and the spectrum $\Sigma(A)$ of $A$ by $\Sigma(A):=\mathbb{C} \backslash \Phi(A)$. The essential spectrum $\Sigma_{\text {ess }}(A)$ of $A$ is $\Sigma_{\text {ess }}(A):=\mathbb{C} \backslash \Phi_{\text {ess }}(A)$, where

$$
\Phi_{\text {ess }}(A)=\{\lambda \in \mathbb{C}: A-\lambda I \text { is Fredholm and } i(A-\lambda I)=0\} .
$$

Theorem 3.2. Let $\sigma$ be the matrix symbol of a pseudo-differential operator $T_{\sigma} \in \Psi^{0}(G)$, and let $d_{\max }:=\lim \sup _{\langle\xi\rangle \rightarrow \infty}\left\{\sup _{x \in G}\|\sigma(x, \xi)\|_{o p}\right\}$. Then for $T_{\sigma}$ operating on $L^{2}(G)$,

$$
\Sigma_{e s s}\left(T_{\sigma}\right) \subseteq\left\{\lambda \in \mathbb{C}:|\lambda| \leq d_{\max }\right\}
$$

Moreover, if $d_{\max }=0$, then $T_{\sigma}$ is a compact operator on $L^{2}(G)$.

In fact, $T_{\sigma}$ is compact if and only if $d_{\max }=0$. Indeed, taking $K=T_{\sigma}$ in Theorem 3.1 shows that if $d_{\text {min }} \neq 0$, then $T_{\sigma}$ is not compact.

In view of (3.2), $d_{\min } \leq d_{\max }$. In case $G=\mathbb{T}^{n}$ is the torus, $d_{\min }=d_{\max }$.

\section{Proof of Theorem 3.1}

First observe that by (3.2), $d_{\min }$ is well-defined; and hence, for every $[\xi] \in \widehat{G}$, there exists $x_{\xi} \in G$ such that

$$
\frac{\left\|\sigma\left(x_{\xi_{n}}, \xi_{n}\right) \sigma\left(x_{\xi_{n}}, \xi_{n}\right)^{*}\right\|_{\min }}{\left\|\sigma\left(x_{\xi_{n}}, \xi_{n}\right)\right\|_{o p}}=\sup _{x \in G} \frac{\left\|\sigma\left(x, \xi_{n}\right) \sigma\left(x, \xi_{n}\right)^{*}\right\|_{\min }}{\left\|\sigma\left(x, \xi_{n}\right)\right\|_{o p}} .
$$

The definition of $d_{\text {min }}$ gives a sequence $\left\{x_{\xi_{n}}, \xi_{n}\right\}_{n=1}^{\infty}$ such that

$$
\lim _{n \rightarrow \infty}\left\langle\xi_{n}\right\rangle=\infty \quad \text { and } \quad \lim _{n \rightarrow \infty} \frac{\left\|\sigma\left(x_{\xi_{n}}, \xi_{n}\right) \sigma\left(x_{\xi_{n}}, \xi_{n}\right)^{*}\right\|_{\min }}{\left\|\sigma\left(x_{\xi_{n}}, \xi_{n}\right)\right\|_{o p}}=d_{\min } .
$$


Define the $L^{2}$-matrix norm of a matrix-valued function $w=w(x) \in \mathbb{C}^{d_{\xi} \times d_{\xi}}$ by

$$
\|w\|_{L^{2}(G)}:=\left(\int_{G}\|w(x)\|_{\mathrm{HS}}^{2} d x\right)^{1 / 2} .
$$

For sufficiently smooth $u \in L^{2}(G)$, define $u_{\xi_{n}}(x):=d_{\xi_{n}}^{-1 / 2} \xi_{n}(x) u\left(x \cdot x_{\xi_{n}}^{-1}\right)$. Then

$$
\begin{aligned}
\left\|u_{\xi_{n}}\right\|_{L^{2}(G)}^{2} & =\int_{G}\left\|u_{\xi_{n}}(x)\right\|_{\mathrm{HS}}^{2} d x=\int_{G} d_{\xi_{n}}^{-1}\left\|\xi_{n}(x) u\left(x \cdot x_{\xi_{n}}^{-1}\right)\right\|_{\mathrm{HS}}^{2} d x \\
& =d_{\xi_{n}}^{-1}\left\|\xi_{n}\right\|_{\mathrm{HS}}^{2}\|u\|_{L^{2}(G)}^{2}=\|u\|_{L^{2}(G)}^{2} .
\end{aligned}
$$

Therefore,

$$
\left\|u_{\xi_{n}}\right\|_{L^{2}(G)}=\|u\|_{L^{2}(G)}=\|\widehat{u}\|_{\ell^{2}(\widehat{G})} .
$$

Now let $\phi \in C^{\infty}(G)$. Then, with $y=x \cdot x_{\xi_{n}}^{-1}$, we have

$$
\begin{aligned}
\int_{G} u_{\xi_{n}}(x) \phi(x) d x & =d_{\xi_{n}}^{-1 / 2} \int_{G} \xi_{n}(y) u(y) \phi\left(y \cdot x_{\xi_{n}}\right) \xi_{n}\left(x_{\xi_{n}}\right) d y \\
& \left.=d_{\xi_{n}}^{-1 / 2} u \widehat{\phi\left(\cdot x_{\xi_{n}}\right.}\right)\left(\xi_{n}^{*}\right) \xi_{n}\left(x_{\xi_{n}}\right),
\end{aligned}
$$

where $\xi_{n}^{*}(x)=\xi_{n}(x)^{*}$. Since $\left\|u \phi\left(\cdot x_{\xi_{n}}\right)\right\|_{L^{2}} \leq C\|u\|_{L^{2}}$ with a constant $C$ independent of $\left.x_{\xi_{n}}, u \widehat{\phi\left(\cdot x_{\xi_{n}}\right.}\right) \in \ell^{2}(\widehat{G})$ uniformly in $x_{\xi_{n}}$. Hence it follows from (4.1) and (2.1) that $\left.d_{\xi_{n}} \| u \widehat{\phi\left(\cdot x_{\xi_{n}}\right.}\right)\left(\xi_{n}^{*}\right) \|_{\mathrm{HS}}^{2} \rightarrow 0$ as $\left\langle\xi_{n}^{*}\right\rangle \rightarrow \infty$. This implies

$$
\left.\left.\left\|\int_{G} u_{\xi_{n}}(x) \phi(x) d x\right\|_{\mathrm{HS}}=\| d_{\xi_{n}}^{-1 / 2} u \widehat{\phi\left(\cdot x_{\xi_{n}}\right.}\right)\left(\xi_{n}^{*}\right) \xi_{n}\left(x_{\xi_{n}}\right)\left\|_{\mathrm{HS}} \leq\right\| u \widehat{\phi\left(\cdot x_{\xi_{n}}\right.}\right)\left(\xi_{n}^{*}\right) \|_{\mathrm{HS}},
$$

so that $u_{\xi_{n}} \rightarrow 0$ as $\left\langle\xi_{n}\right\rangle \rightarrow \infty$ weakly. Hence, for a compact operator $K$,

$$
\left\|K u_{\xi_{n}}\right\|_{L^{2}(G)} \rightarrow 0 \quad \text { as }\left\langle\xi_{n}\right\rangle \rightarrow \infty .
$$

Then, by compactness, for $\epsilon>0$ and sufficiently large $n$,

$$
\left\|K u_{\xi_{n}}\right\|_{L^{2}(G)} \leq \epsilon\left\|u_{\xi_{n}}\right\|_{L^{2}(G)}=\epsilon\|u\|_{L^{2}(G)},
$$

where $u$ is fixed and $\left\|K u_{\xi_{n}}\right\|_{L^{2}(G)}=\left(\int_{G}\left\|K u_{\xi_{n}}(x)\right\|_{\mathrm{HS}}^{2} d x\right)^{1 / 2}$.

Lemma 4.1. $\left\|u_{\xi_{n}} \sigma\left(\cdot, \xi_{n}\right)-T_{\sigma} u_{\xi_{n}}\right\|_{L^{2}(G)} \rightarrow 0$ as $\left\langle\xi_{n}\right\rangle \rightarrow \infty$, where $T_{\sigma} u_{\xi_{n}}:=$ $\left(T_{\sigma}\left(u_{\xi_{n}}\right)_{i j}\right)_{1 \leq i, j \leq d_{\xi_{n}}} \in \mathbb{C}^{d_{\xi_{n}} \times d_{\xi_{n}}}$.

We postpone the proof of Lemma 4.1 and continue with the proof of Theorem 3.1.

Let us fix nonzero $u \in C^{\infty}(G)$. Then for each $\epsilon>0$, there exists $N(u)$ such that for all $n \geq N(u)$,

$$
\left\|u_{\xi_{n}} \sigma\left(\cdot, \xi_{n}\right)\right\|_{L^{2}(G)}-\left\|T_{\sigma} u_{\xi_{n}}\right\|_{L^{2}(G)} \leq \epsilon\|u\|_{L^{2}(G)}
$$


for sufficiently large $\left\langle\xi_{n}\right\rangle$. Now, since $\sigma$ satisfies (2.3) with $\alpha=0$, the derivatives of $\sigma$ in $x$ of are uniformly bounded; hence, for each $\epsilon>0$, there exists an open neighborhood $V \subseteq G$ of $e$ such that $\left\|\sigma\left(x, \xi_{n}\right)-\sigma\left(x_{\xi_{n}}, \xi_{n}\right)\right\|_{o p}<\epsilon$ for all $x \cdot x_{\xi_{n}}^{-1} \in V$. Let now $u \in C^{\infty}(G)$ be such that $u(x)=0$ for all $x \notin V$. Then $u_{\xi_{n}}(x)=0$ for all $x \notin x_{\xi_{n}} V$, i.e., for all $x$ such that $x \cdot x_{\xi_{n}}^{-1} \notin V$. Hence

$$
\begin{aligned}
& \frac{\left\|u_{\xi_{n}} \sigma\left(x_{\xi_{n}}, \xi_{n}\right) \sigma\left(x_{\xi_{n}}, \xi_{n}\right)^{*}\right\|_{L^{2}(G)}}{\left\|\sigma\left(x_{\xi_{n}}, \xi_{n}\right)\right\|_{o p}}-\left\|u_{\xi_{n}} \sigma\left(\cdot, \xi_{n}\right)\right\|_{L^{2}(G)} \\
& \leq\left\|u_{\xi_{n}} \sigma\left(x_{\xi_{n}}, \xi_{n}\right)\right\|_{L^{2}(G)}-\left\|u_{\xi_{n}} \sigma\left(\cdot, \xi_{n}\right)\right\|_{L^{2}(G)} \\
& \leq\left\|u_{\xi_{n}} \sigma\left(x_{\xi_{n}}, \xi_{n}\right)-u_{\xi_{n}} \sigma\left(\cdot, \xi_{n}\right)\right\|_{L^{2}(G)} \\
& \quad \leq\left(\int_{x_{\xi_{n}} V}\left\|\sigma\left(x, \xi_{n}\right)-\sigma\left(x_{\xi_{n}}, \xi_{n}\right)\right\|_{o p}^{2}\left\|u_{\xi_{n}}(x)\right\|_{\mathrm{HS}}^{2} d x\right)^{1 / 2} \\
& \leq \epsilon\left(\int_{x_{\xi_{n} V}}\left\|u_{\xi_{n}}(x)\right\|_{\mathrm{HS}}^{2}\right)^{1 / 2}=\epsilon\left\|u_{\xi_{n}}\right\|_{L^{2}(G)}=\epsilon\|u\|_{L^{2}(G)},
\end{aligned}
$$

the last inequality following from (4.1). Therefore,

$$
\begin{aligned}
\|u\|_{L^{2}(G)}\left\|T_{\sigma}-K\right\|_{\mathscr{L}\left(L^{2}(G)\right)} & =\left\|u_{\xi_{n}}\right\|_{L^{2}(G)}\left\|T_{\sigma}-K\right\|_{\mathscr{L}\left(L^{2}(G)\right)} \\
& \geq\left\|\left(T_{\sigma}-K\right) u_{\xi_{n}}\right\|_{L^{2}(G)} \\
& \geq\left\|T_{\sigma} u_{\xi_{n}}\right\|_{L^{2}(G)}-\left\|K u_{\xi_{n}}\right\|_{L^{2}(G)} \\
& \geq\left\|u_{\xi_{n}} \sigma\left(\cdot, \xi_{n}\right)\right\|_{L^{2}(G)}-2 \epsilon\|u\|_{L^{2}(G)} \\
& \geq \frac{\left\|u_{\xi_{n}} \sigma\left(x_{\xi_{n}}, \xi_{n}\right) \sigma\left(x_{\xi_{n}}, \xi_{n}\right)^{*}\right\|_{L^{2}(G)}-3 \epsilon\|u\|_{L^{2}(G)},}{\left\|\sigma\left(x_{\xi_{n}}, \xi_{n}\right)\right\|_{o p}}
\end{aligned}
$$

the last inequalities following from (4.2) and (4.3), respectively. Moreover, since $\sigma\left(x_{\xi_{n}}, \xi_{n}\right) \sigma\left(x_{\xi_{n}}, \xi_{n}\right)^{*}$ is normal, there exist a unitary matrix $U$ such that

$$
\sigma\left(x_{\xi_{n}}, \xi_{n}\right) \sigma\left(x_{\xi_{n}}, \xi_{n}\right)^{*}=U \Lambda U^{*}
$$

where

$$
\Lambda=\left[\begin{array}{cccc}
\lambda_{11} & 0 & \ldots & 0 \\
0 & \lambda_{22} & \ldots & 0 \\
\vdots & \vdots & \ddots & \vdots \\
0 & 0 & \ldots & \lambda_{d_{\tilde{\xi}} d_{\tilde{\xi}}}
\end{array}\right] .
$$

Here, $\lambda_{i i}\left(x_{\xi_{n}}, \xi_{n}\right)$ are the eigenvalues of $\sigma\left(x_{\xi_{n}}, \xi_{n}\right) \sigma\left(x_{\xi_{n}}, \xi_{n}\right)^{*}$.

Let $\lambda_{m m}\left(x_{\xi_{n}}, \xi_{n}\right)=\min _{1 \leq i \leq d_{\xi}} \lambda_{i i}\left(x_{\xi_{n}}, \xi_{n}\right)$. Now $U \Lambda U^{*}$ is symmetric, and $\lambda_{m m}^{-1}\left(x_{\xi_{n}}, \xi_{n}\right)$ is the maximum eigenvalue of $U \Lambda^{-1} U^{*}$, i.e.,

$$
\left\|U \Lambda^{-1} U^{*}\right\|_{o p}=\lambda_{m m}^{-1}\left(x_{\xi_{n}}, \xi_{n}\right) .
$$


Hence $\left\|u_{\xi_{n}}(x) U \Lambda U^{*}\right\|_{\mathrm{HS}} \geq \lambda_{m m}\left(x_{\xi_{n}}, \xi_{n}\right)\left\|u_{\xi_{n}}(x) U \Lambda U^{*}\left(U \Lambda U^{*}\right)^{-1}\right\|_{\mathrm{HS}}$, from which it follows that

$$
\left\|u_{\xi_{n}}(x) U \Lambda U^{*}\right\|_{\mathrm{HS}}^{2} \geq \lambda_{m m}^{2}\left\|u_{\xi_{n}}(x)\right\|_{\mathrm{HS}}^{2} .
$$

Using (4.4) and (4.5), we obtain

$$
\begin{aligned}
\|u\|_{L^{2}(G)}\left\|T_{\sigma}-K\right\|_{\mathscr{L}\left(L^{2}(G)\right)} & \geq \frac{\left(\int_{G}\left\|u_{\xi_{n}} \sigma\left(x_{\xi_{n}}, \xi_{n}\right) \sigma\left(x_{\xi_{n}}, \xi_{n}\right)^{*}\right\|_{\mathrm{HS}}^{2} d x\right)^{1 / 2}}{\left\|\sigma\left(x_{\xi_{n}}, \xi_{n}\right)\right\|_{o p}}-3 \epsilon\|u\|_{L^{2}(G)} \\
& \geq \frac{\left(\lambda_{m m}^{2} \int_{G}\left\|u_{\xi_{n}}(x)\right\|_{\mathrm{HS}}^{2} d x\right)^{1 / 2}}{\left\|\sigma\left(x_{\xi_{n}}, \xi_{n}\right)\right\|_{o p}}-3 \epsilon\|u\|_{L^{2}(G)} \\
& =\frac{\lambda_{m m}\left\|u_{\xi_{n}}\right\|_{L^{2}(G)}}{\left\|\sigma\left(x_{\xi_{n}}, \xi_{n}\right)\right\|_{o p}}-3 \epsilon\|u\|_{L^{2}(G)} \\
& =\frac{\lambda_{m m}}{\left\|\sigma\left(x_{\xi_{n}}, \xi_{n}\right)\right\|_{o p}}\|u\|_{L^{2}(G)}-3 \epsilon\|u\|_{L^{2}(G)} \\
& =\left(\frac{\lambda_{m m}\left(x_{\xi_{n}}, \xi_{n}\right)}{\left\|\sigma\left(x_{\xi_{n}}, \xi_{n}\right)\right\|_{o p}}-3 \epsilon\right)\|u\|_{L^{2}(G)} .
\end{aligned}
$$

Now, for sufficiently large $\left\langle\xi_{n}\right\rangle \rightarrow,\|u\|_{L^{2}(G)}\left\|T_{\sigma}-K\right\|_{*} \geq\left(d_{\min }-3 \epsilon\right)\|u\|_{L^{2}(G)}$, i.e.,

$$
\left\|T_{\sigma}-K\right\|_{*} \geq d_{\min }-3 \epsilon \text { for all } \epsilon>0
$$

from which it follows that $\left\|T_{\sigma}-K\right\|_{*} \geq d_{\min }$. This completes the proof of Theorem 3.1 .

Proof of Lemma 4.1. For $x, z \in G$, let $R(x, z):=\sum_{[\xi] \in \widehat{G}} d_{\xi} \operatorname{Tr}(\sigma(x, \xi) \xi(z))$. Thus

$$
\begin{aligned}
T_{\sigma} u(x) & =\sum_{[\xi] \in \widehat{G}} d_{\xi} \operatorname{Tr}(\xi(x) \sigma(x, \xi) \widehat{u}(\xi)) \\
& =\int_{G} \sum_{[\xi] \in \widehat{G}} d_{\xi} \operatorname{Tr}\left(\xi(x) \sigma(x, \xi) \xi^{*}(y)\right) u(y) d y \\
& =\int_{G} \sum_{[\xi] \in \widehat{G}} d_{\xi} \operatorname{Tr}\left(\sigma(x, \xi) \xi\left(y^{-1} x\right)\right) u(y) d y \\
& =\int_{G} R\left(x, y^{-1} x\right) u(y) d y \\
& =\int_{G} R(x, z) u\left(x z^{-1}\right) d z,
\end{aligned}
$$

where $z=y^{-1} x$. From the definition of $u_{\xi_{n}}(x)=d_{\xi_{n}}^{-1 / 2} \xi_{n}(x) u\left(x x_{\xi_{n}}^{-1}\right)$ and (4.7), we 
obtain

$$
\begin{aligned}
T_{\sigma} u_{\xi_{n}}(x) & =d_{\xi_{n}}^{-1 / 2} \int_{G} R(x, z) \xi_{n}\left(x z^{-1}\right) u\left(x z^{-1} x_{\xi_{n}}^{-1}\right) d z \\
& =d_{\xi_{n}}^{-1 / 2} \int_{G} R(x, z) \xi_{n}(x) u\left(x z^{-1} x_{\xi_{n}}^{-1}\right) \xi_{n}^{*}(z) d z
\end{aligned}
$$

Letting

$$
v_{\xi_{n}}^{x}\left(z^{-1}\right):=v_{\xi_{n}}\left(x z^{-1}\right):=d_{\xi_{n}}^{-1 / 2} \xi_{n}(x) u\left(x z^{-1} x_{\xi_{n}}^{-1}\right)
$$

we have

$$
T_{\sigma} u_{\xi_{n}}(x)=\int_{G} R(x, z) v_{\xi_{n}}^{x}\left(z^{-1}\right) \xi_{n}^{*}(z) d z .
$$

For a given collection $\Delta_{1}, \ldots, \Delta_{m}$ of strongly admissible difference operators with corresponding functions $q_{1}, \ldots, q_{m} \in C^{\infty}(G)$ satisfying $\Delta_{j} \widehat{f}(\xi)=\widehat{q_{j} f}(\xi)$, we have the Taylor expansion formula (see [RT10, RTW10])

$$
u(x)=u(e)+\sum_{|\alpha|=1}^{N-1} \frac{1}{\alpha !} q^{\alpha}\left(x^{-1}\right) \partial^{(\alpha)} u(e)+\mathcal{O}\left(h(x)^{N}\right),
$$

where $h(x)$ is the geodesic distance from $x$ and $e, \partial_{x}^{(\alpha)}$ are left-invariant differential operators on $G$, and $q^{\alpha}(x)=q_{1}(x)^{\alpha_{1}} \cdots q_{m}(x)^{\alpha_{m}}$. For $u$ sufficiently smooth, the Taylor expansion formula gives

$$
v_{\xi_{n}}^{x}\left(z^{-1}\right)=v_{\xi_{n}}^{x}(e)+\sum_{|\alpha|=1}^{N-1} \frac{1}{\alpha !} q^{\alpha}(z) \partial^{(\alpha)} v_{\xi_{n}}^{x}(e)+\mathcal{O}\left(h(z)^{N}\right) .
$$

By the left-invariance of $\partial_{x}^{(\alpha)}$, we obtain

$$
v_{\xi_{n}}\left(x z^{-1}\right)=v_{\xi_{n}}(x)+\sum_{|\alpha|=1}^{N-1} \frac{1}{\alpha !} q^{\alpha}(z) \partial^{(\alpha)} v_{\xi_{n}}(x)+\mathcal{O}\left(h(z)^{N}\right) .
$$

Using (4.10) and (4.11), we can now write

$$
\begin{aligned}
T_{\sigma} u_{\xi_{n}}(x)=\int_{G} R(x, z) v_{\xi_{n}}(x) \xi_{n}^{*}(z) d z & +\int_{G} R(x, z) \sum_{|\alpha|=1}^{N-1} \frac{1}{\alpha !} q^{\alpha}(z) \partial^{(\alpha)} v_{\xi_{n}}(x) \xi_{n}^{*}(z) d z \\
& +\int_{G} R(x, z) \mathcal{O}\left(h(z)^{N}\right) \xi_{n}^{*}(z) d z .
\end{aligned}
$$

Defining

$$
\begin{aligned}
I_{1} & :=\int_{G} R(x, z) v_{\xi_{n}}(x) \xi_{n}^{*}(z) d z, \\
I_{2} & :=\int_{G} R(x, z) \sum_{|\alpha|=1}^{N-1} \frac{1}{\alpha !} q^{\alpha}(z) \partial^{(\alpha)} v_{\xi_{n}}(x) \xi_{n}^{*}(z) d z,
\end{aligned}
$$


and

$$
I_{3}:=\int_{G} R(x, z) \mathcal{O}\left(h(z)^{N}\right) \xi_{n}^{*}(z) d z
$$

we have

$$
\begin{aligned}
I_{1} & =\int_{G} R(x, z) v_{\xi_{n}}(x) \xi_{n}^{*}(z) d z=v_{\xi_{n}}(x) \sigma\left(x, \xi_{n}\right) \\
& =d_{\xi_{n}}^{-1 / 2} \xi_{n}(x) u\left(x x_{\xi_{n}}^{-1}\right) \sigma\left(x, \xi_{n}\right)=u_{\xi_{n}}(x) \sigma\left(x, \xi_{n}\right), \\
I_{2} & =\int_{G} R(x, z) \partial^{(\alpha)} v_{\xi_{n}}(x) \xi_{n}^{*}(z) \sum_{|\alpha|=1}^{N-1} \frac{1}{\alpha !} q^{\alpha}(z) d z \\
& =\sum_{|\alpha|=1}^{N-1} \frac{1}{\alpha !} \partial^{(\alpha)} v_{\xi_{n}}(x) \int_{G} R(x, z) \xi_{n}^{*}(z) q^{\alpha}(z) d z \\
& =\sum_{|\alpha|=1}^{N-1} \frac{1}{\alpha !} \partial^{(\alpha)} v_{\xi_{n}}(x)\left(\mathcal{F} q^{\alpha} \mathcal{F}^{-1} \sigma\right)\left(\xi_{n}\right) \\
& =\sum_{|\alpha|=1}^{N-1} \frac{1}{\alpha !} \partial^{(\alpha)} v_{\xi_{n}}(x) \Delta_{q^{\alpha}} \sigma\left(x, \xi_{n}\right),
\end{aligned}
$$

and

$$
\begin{aligned}
I_{3} & =\int_{G} R(x, z) \mathcal{O}\left(h(z)^{N}\right) \xi_{n}^{*}(z) d z=\int_{G} R(x, z) q^{N}(z) \xi_{n}^{*}(z) d z \\
& =\Delta_{q^{N}} \sigma\left(x, \xi_{n}\right),
\end{aligned}
$$

where the matrix-valued function $q^{N}:=\mathcal{O}\left(h(x)^{N}\right)$ vanishes at $e$ to order $N$. Hence

$$
T_{\sigma} u_{\xi_{n}}(x)-u_{\xi_{n}}(x) \sigma\left(x, \xi_{n}\right)=\sum_{|\alpha|=1}^{N-1} \frac{1}{\alpha !} \partial^{(\alpha)} v_{\xi_{n}}(x) \Delta_{q^{\alpha}} \sigma\left(x, \xi_{n}\right)+\Delta_{q^{N}} \sigma\left(x, \xi_{n}\right) .
$$

Defining $T_{N}^{1}:=\sum_{|\alpha|=1}^{N-1} \frac{1}{\alpha !} \partial^{(\alpha)} v_{\xi_{n}}(x) \Delta_{q^{\alpha}} \sigma\left(x, \xi_{n}\right)$ and $T_{N}^{2}:=\Delta_{q^{N}} \sigma\left(x, \xi_{n}\right)$, we obtain

$$
\left\|T_{N}^{1}(x)\right\|_{\mathrm{HS}} \leq\left\|\Delta_{q^{\alpha}} \sigma\left(x, \xi_{n}\right)\right\|_{o p} \sum_{0<|\alpha| \leq N} \frac{1}{\alpha !}\left\|\partial_{z}^{(\alpha)} v_{\xi_{n}}^{x}(x)\right\|_{\mathrm{HS}},
$$

where $z \in G$. Using (4.9) and (2.3), we have

$$
\begin{aligned}
\left\|T_{N}^{1}(x)\right\|_{\mathrm{HS}} & \leq C\left(\sum_{0<|\alpha| \leq N}\left|\tilde{\partial}_{z}^{(\alpha)} u\left(x \cdot x_{\xi_{n}-1}\right)\right|\right) d_{\xi_{n}}^{-1 / 2}\left\|\xi_{n}(x)\right\|_{\mathrm{HS}}\left\langle\xi_{n}\right\rangle^{-|\alpha|} \\
& \leq C \sum_{0<|\alpha| \leq N}\|u\|_{H^{|\alpha|}}\left\langle\xi_{n}\right\rangle^{-|\alpha|} \leq C^{\prime}\left\langle\xi_{n}\right\rangle^{-1}
\end{aligned}
$$


for some operator $\widetilde{\partial}_{z}^{(\alpha)}$, where $N$ is fixed, $1 \leq|\alpha| \leq N$, and $\|\cdot\|_{H^{|\alpha|}}$ is the Sobolev norm. Similarly, $\left\|T_{N}^{2}\right\|_{\mathrm{HS}} \leq C\left\langle\xi_{n}\right\rangle^{-N}$.

Therefore, $\left\|T_{N}^{1}(x)\right\|_{\mathrm{HS}} \rightarrow 0$ and $\left\|T_{N}^{2}(x)\right\|_{\mathrm{HS}} \rightarrow 0$ as $\left\langle\xi_{n}\right\rangle \rightarrow \infty$ for all $x \in G$, which gives $\left\|T_{N}^{1}\right\|_{L^{2}(G)}^{2}=\int_{G}\left\|T_{N}^{1}(x)\right\|_{\mathrm{HS}}^{2} d x \rightarrow 0$ as $\left\langle\xi_{n}\right\rangle \rightarrow \infty$. Similarly, $\left\|T_{N}^{2}\right\|_{L^{2}(G)} \rightarrow 0$. This implies $\left\|T_{\sigma} u_{\xi_{n}}-u_{\xi_{n}} \sigma\left(\cdot, \xi_{n}\right)\right\|_{L^{2}(G)} \rightarrow 0$ as $\left\langle\xi_{n}\right\rangle \rightarrow \infty$. We complete the proof by observing that it suffices to take $N=1$ in the above argument.

Remark 4.2. With the same proof (and with $N=1$ ), we have the following extension of the Gohberg Lemma without the assumption that the operator $T_{\sigma}$ belongs to $\Psi^{0}(G)$.

Let $T_{\sigma}: L^{2}(G) \rightarrow L^{2}(G)$ be a bounded operator whose matrix symbol $\sigma(x, \xi)$ satisfies

$$
\|\sigma(x, \xi)\|_{o p} \leq C, \quad\left\|\partial_{x} \sigma(x, \xi)\right\|_{o p} \leq C, \quad\left\|\Delta_{q} \sigma(x, \xi)\right\|_{o p} \leq C\langle\xi\rangle^{-\rho}
$$

for some $\rho>0$ for all $q \in C^{\infty}(G)$ with $q(e)=0$ and all $x \in G$ and $[\xi] \in \widehat{G}$. Then the conclusion of the Gohberg Lemma in Theorem 3.1 remains true, namely, the estimate (3.1) holds for all compact operators $K$ on $L^{2}(G)$.

\section{Proof of Theorem 3.2}

We first recall the following theorem, which gives another (equivalent) definition of Fredholm operators.

Theorem 5.1 (Atkinson's Theorem [Atk51]). Let A be a closed linear operator from a complex Banach space $X$ into a complex Banach space $Y$ with a dense domain $D(A)$. Then $A$ is Fredholm if and only if there exist a bounded linear operator $B: Y \rightarrow X$, a compact operator $K_{1}: X \rightarrow X$ and a compact operator $K_{2}: Y \rightarrow Y$ such that $B A=I+K_{1}$ on $D(A)$ and $A B=I+K_{2}$ on $Y$.

Recall that the Wolf spectrum $\Sigma_{w}(A)$ of $A$ is defined by $\Sigma_{w}(A):=\mathbb{C} \backslash \Phi_{w}(A)$, where $\Phi_{w}(A)=\{\lambda \in \mathbb{C}: A-\lambda I$ is Fredholm $\}$. Clearly, $\Sigma_{w}(A) \subseteq \Sigma_{\text {ess }}(A) \subseteq$ $\Sigma(A)$.

Proof of Theorem 3.2. Let $\lambda \in \mathbb{C}$ be such that $|\lambda|>d_{\max }$. Then there exists $\epsilon>0$ such that $|\lambda|>d_{\max }+\epsilon$. Now, by the definition of $d_{\max }$ in Theorem 3.2, there exists $R>0$ such that for all $\langle\xi\rangle \geq R$,

$$
\sup \left\{\sup \|\sigma(x, \xi)\|_{o p}\right\} \leq\left(d_{\max }+\epsilon / 2\right) .
$$

$\langle\xi\rangle \geq R \quad x \in G$ 
Then for $\langle\xi\rangle \geq R$,

$$
\begin{aligned}
& \left\|(\sigma(x, \xi)-\lambda I)^{-1}\right\|_{o p} \leq \sum_{k=1}^{\infty} \lambda^{-k-1}\left\|\sigma(x, \xi)^{k}\right\|_{o p} \\
& \quad \leq \sum_{k=1}^{\infty} \frac{\left(d_{\max }+\epsilon / 2\right)^{k}}{|\lambda|^{k+1}} \leq \sum_{k=1}^{\infty} \frac{\left(d_{\max }+\epsilon / 2\right)^{k}}{\left(d_{\max }+\epsilon\right)^{k+1}} \leq C \sum_{k=1}^{\infty} \frac{\left(d_{\max }+\epsilon / 2\right)^{k}}{\left(d_{\max }+\epsilon\right)^{k}}<\infty .
\end{aligned}
$$

It follows from (2.4) that the operator $T_{\sigma}-\lambda I: L^{2}(G) \rightarrow L^{2}(G)$ is elliptic and hence a Fredholm operator; see, e.g., [Hör07, Section 19.5]. Thus

$$
\left\{\lambda \in \mathbb{C}:|\lambda|>d_{\max }\right\} \subseteq \Phi_{w}\left(T_{\sigma}\right),
$$

which implies $\Sigma_{w}\left(T_{\sigma}\right) \subseteq\left\{\lambda \in \mathbb{C}:|\lambda| \leq d_{\max }\right\}$. Since $\left\{\lambda \in \mathbb{C}:|\lambda|>d_{\max }\right\}$ is a connected component of $\Phi_{w}\left(T_{\sigma}\right), i\left(T_{\sigma}-\lambda I\right)$ is constant on $\left\{\lambda \in \mathbb{C}:|\lambda|>d_{\max }\right\}$. Now, again $\Phi\left(T_{\sigma}\right) \cap\left\{\lambda \in \mathbb{C}:|\lambda|>d_{\max }\right\} \neq \varnothing$. Therefore $i\left(T_{\sigma}-\lambda I\right)=0$ for all $\left\{\lambda \in \mathbb{C}:|\lambda|>d_{\text {max }}\right\}$, which implies $\Sigma_{\text {ess }}\left(T_{\sigma}\right) \subseteq\left\{\lambda \in \mathbb{C}:|\lambda| \leq d_{\max }\right\}$, completing the proof of (3.3).

We now turn to the proof of the last part of Theorem 3.2. Let $\mathscr{L}\left(L^{2}(G)\right)$ and be the $C^{*}$ algebra of bounded linear operators on $L^{2}(G)$ and $\mathscr{K}\left(L^{2}(G)\right)$ the ideal of compact operators on $L^{2}(G)$. Define multiplication on $\mathscr{L}\left(L^{2}(G)\right) / \mathscr{K}\left(L^{2}(G)\right)$ by $[A][B]:=[A B]$ and involution by $[A]^{*}:=\left[A^{*}\right]$. These operations make $\mathscr{L}\left(L^{2}(G)\right) / \mathscr{K}\left(L^{2}(G)\right)$ into a $*$-algebra, known as the Calkin algebra. For $[A],[B] \in \mathscr{L}\left(L^{2}(G)\right) / \mathscr{K}\left(L^{2}(G)\right),[A]=[B]$ if and only if $A-B \in \mathscr{K}\left(L^{2}(G)\right)$. The norm $\|\cdot\|_{C}$ in $\mathscr{L}\left(L^{2}(G)\right) / \mathscr{K}\left(L^{2}(G)\right)$ is given by

$$
\|[A]\|_{C}:=\inf _{K \in \mathscr{K}\left(L^{2}(G)\right)}\|A-K\|_{\mathscr{L}\left(L^{2}(G)\right)}, \quad[A] \in \mathscr{L}\left(L^{2}(G)\right) / \mathscr{K}\left(L^{2}(G)\right) .
$$

Using the Calkin algebra, we can reformulate (3.1) as $\left\|\left[T_{\sigma}\right]\right\|_{C} \geq d_{\min }$.

Assume now that $d_{\max }=0$ and observe that $T_{\sigma}$ is compact if and only if [ $\left.T_{\sigma}\right]=$ 0 in the Calkin algebra. Observe also that $T_{\sigma}$ is essentially normal on $L^{2}(G)$, i.e., $T_{\sigma} T_{\sigma}^{*}-T_{\sigma}^{*} T_{\sigma}$ is compact. Indeed, $T_{\sigma} T_{\sigma}^{*}-T_{\sigma}^{*} T_{\sigma}$ is an operator of order -1 , so its compactness follows from the compactness of the embedding $H^{1} \hookrightarrow L^{2}$. Consequently, [ $\left.T_{\sigma}\right]$ is normal in $\mathscr{L}\left(L^{2}(G)\right) / \mathscr{K}\left(L^{2}(G)\right)$; and, therefore, $r\left(T_{\sigma}\right)=$ $\left\|\left[T_{\sigma}\right]\right\|_{C}$, where $r\left(T_{\sigma}\right)$ is the spectral radius of $\left[T_{\sigma}\right]$. On the other hand, by (3.3), $\Sigma_{e s s}\left(T_{\sigma}\right) \subset\{0\}$, which implies that $T_{\sigma}-\lambda I$ is Fredholm for $\lambda \neq 0$. In light of Atkinson's Theorem, this implies that there exists a bounded operator $B$ such that $\left(T_{\sigma}-\lambda I\right) B=I+K$, where $K$ is a compact operator; i.e., $\left[\left(T_{\sigma}-\lambda I\right)\right]$ is invertible for $\lambda \neq 0$, which implies that $\lambda \notin \Sigma\left(\left[T_{\sigma}\right]\right)$ if $\lambda \neq 0$. Thus $\Sigma\left(\left[T_{\sigma}\right]\right) \subseteq\{0\}$, from which it follows that $\left\|\left[T_{\sigma}\right]\right\|_{C}=r\left(T_{\sigma}\right)=0$; and so $\left[T_{\sigma}\right]=0$. Hence $T_{\sigma}$ is compact. 


\section{REFERENCES}

[Atk51] F. V. Atkinson, The normal solvability of linear equations in normed spaces, Mat. Sb. (N. S.) 28 (1951), 3-14.

[DR13a] J. Delgado and M. Ruzhansky, $L^{p}$-nuclearity, traces, and Grothendieck-Lidskii formula on compact Lie groups, J. Math. Pures Appl. (9) 102 (2014), 153-172.

[DR13b] J. Delgado and M. Ruzhansky. Schatten classes and traces on compact Lie groups, arXiv:1303.3914[ma.FA].

[Goh60] I. C. Gohberg, On the theory of multidimensional singular integral equations, Soviet Math. Dokl. 1 (1960), 960-963.

[Hör07] L. Hörmander, The Analysis of Linear Partial Differential Operators, III, Springer, Berlin, 2007.

[Mol11] S. Molahajloo, A characterization of compact pseudo-differential operators on $\mathbb{S}^{1}$, in Pseudo-differential Operators: Analysis, Applications and Computations, Birkhäuser/ Springer Basel AG, Basel, 2011, pp. 25-29.

[MW10] S. Molahajloo and M. W. Wong, Ellipticity, Fredholmness and spectral invariance of pseudo-differential operators on $\mathbb{S}^{1}$, J. Pseudo-Differ. Oper. Appl. 1 (2010), 183-205.

[Pir11] M. Pirhayati, Spectral theory of pseudo-differential operators on $\mathbb{S}^{1}$, in Pseudo-differential Operators: Analysis, Applications and Computations, Birkhäuser/Springer Basel AG, Basel, 2011, pp. 15-23.

[RT10] M. Ruzhansky and V. Turunen, Pseudo-differential Operators and Symmetries. Background Analysis and Advanced Topics, Birkhäuser Verlag, Basel, 2010.

[RT12] M. Ruzhansky and V. Turunen, Global quantization of pseudo-differential operators on compact Lie groups, SU(2), 3-sphere, and homogeneous spaces, Int. Math. Res. Not. IMRN 2013 (2013), 2439-2496.

[RTW10] M. Ruzhansky, V. Turunen, and J. Wirth, Hörmander class of pseudo-differential operators on compact Lie groups and global hypoellipticity, J. Fourier Anal. Appl. 20 (2014), 476499.

[Sch88] E. Schrohe, Complex powers on noncompact manifolds and manifolds with singularities, Math. Ann. 281 (1988), 393-409.

[See65] R. T. Seeley, Integro-differential operators on vector bundles, Trans. Amer. Math. Soc. 117 (1965), 167-204.

Aparajita Dasgupta AND Michael Ruzhansky

Department of MATHEMATICS

IMPERIAL COLLEGE LONDON

180 QUEEN's GATE, LONDON SW7 2AZ UK

email: a.dasgupta@imperial.ac.uk, m.ruzhansky@imperial.ac.uk

(Received June 1, 2013) 